\title{
Processing, Microstructure and Mechanical Properties of Ti6Al4V Particles-Reinforced Mg Matrix Composites
}

\author{
X. M. Wang ${ }^{1}$ X. J. Wang ${ }^{1}$ X. S. $\mathrm{Hu}^{1} \cdot$ K. Wu ${ }^{1}$ M. Y. Zheng ${ }^{1}$
}

Received: 19 May 2016/Revised: 6 June 2016/Published online: 17 August 2016

(C) The Chinese Society for Metals and Springer-Verlag Berlin Heidelberg 2016

\begin{abstract}
Novel Ti6Al4V particles-reinforced AZ91 Mg matrix composites were successfully fabricated by stir casting method. The stirring time in semisolid condition directly affected the particle distribution and the quality of the ingots. Furthermore, the optimal speed of the heating and the liquid stirring could overcome particle settlement caused by the density difference between the matrix and the particles. Ti6Al4V particles distributed uniformly in the composites with different particle contents. The average grain size decreased with the increase in the particle contents. The Ti6Al4V particles bonded pretty well with the alloy matrix. In addition, there were some interfacial reactions in the composites. There were rod-like $\mathrm{Al}_{3} \mathrm{Ti}$ phases at the interface. The precipitates extended from the particle surface to the matrix, and they might improve the interfacial bonding strength. The ultimate tensile strength, yield strength and elastic modulus were enhanced as the particle contents increased, and the elongation was much better than that of the same matrix material reinforced with $\mathrm{SiC}$ particles. Thus, the novel composites exhibit better comprehensive mechanical properties.
\end{abstract}

\section{KEY WORDS: Magnesium matrix composites; Ti6Al4V particles; Stir casting; Microstructure; Mechanical} properties

\section{Introduction}

Magnesium alloys have obtained increasing attention for applications in the aerospace and automotive industry in recent years due to their light weight [1,2], high specific strength and stiffness [3, 4], excellent damping capacity [5] and good cast characteristics [6]. The use of these alloys, however, has been restricted by their relatively low absolute strength and elastic modulus when they are compared to other metallic structural materials $[7,8]$. These limitations are often overcome by adding quite a few stiff and

Available online at http://link.springer.com/journal/40195

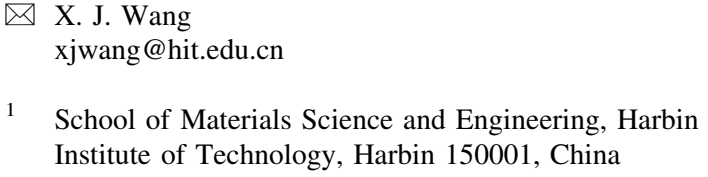

1 School of Materials Science and Engineering, Harbin Institute of Technology, Harbin 150001, China

strong reinforcements into magnesium alloys [9, 10]. Conventionally, magnesium alloys are reinforced with hard ceramic particle reinforcements such as $\mathrm{SiC}, \mathrm{TiC}, \mathrm{TiB}$, $\mathrm{Al}_{2} \mathrm{O}_{3}, \mathrm{Y}_{2} \mathrm{O}_{3}, \mathrm{TiO}_{2}$ [11]. Although ceramic reinforcements usually improve strength and stiffness, they also cause a considerable decrease in the elongation $[12,13]$. The poor elongation of magnesium matrix composites reinforced with ceramic particles may be primarily attributed to the considerable difference between magnesium matrix and ceramic reinforcements, including the crystal structure, the coefficient of thermal expansion and the elastic modulus [14]. Moreover, the ceramic particles are nondeformable, which further accelerates degradation of the properties of the composites. However, titanium with high strength and elastic modulus can exactly overcome the demerits of ceramic particles and surpass such pitfalls, which is a more promising selection of reinforcement for magnesium matrix composites. Additional benefit of titanium could be ascribed to be deformable; in this way that part of the 
applied stress might be transferred to the titanium reinforcement during plastic deformation [8]. Compared to the ceramic particles, the uppermost advantage is that titanium has a better wettability with liquid metal, which aids in the interfacial integrity between the matrix and the reinforcement [15]. In the previous studies, pure titanium [8, 11], titanium alloy $[14,16]$ and hybrid reinforcements containing titanium particulate-reinforced magnesium matrix composites [17-19] were fabricated via powder metallurgy route, whereas the conventional solid-state sintering process could not sufficiently exert the merit of good wettability between titanium and magnesium [20, 21]. In such cases, not only was the increase in the strength limited, but the ductility was also sharply reduced. Besides, there are some researchers who utilized disintegrated melt deposition technology (DMD) to synthesize magnesium matrix composites reinforced with titanium particulates [22, 23] or hybrid reinforcements containing titanium particulates $[24,25]$. The microstructure of the composite materials exhibited strong $\mathrm{Mg}$-Ti interfacial bonding and presence of minimal porosity, suggesting that it was valid for strengthening the interfacial integrity between the $\mathrm{Mg}$ matrix and the Ti particles to use the liquid-state producing approach. In comparison with DMD method, stir casting is more productive and economical [26]. In this regard, it is more competitive for choosing stir casting to fabricate titanium particle-reinforced magnesium matrix composites than other methods. So far, few studies have been carried out on magnesium matrix composites reinforced with titanium alloy particles via stir casting method. What is more, the effects of solidification process on the distribution of titanium reinforcements and the interfacial integrity between magnesium matrix and titanium alloy particles need to be further investigated.

Thus, the primary aim of this article was to investigate the processing, microstructure and mechanical properties of Ti6Al4V particles-reinforced magnesium matrix composites fabricated by stir casting method. Especially, the interface between the magnesium matrix and titanium alloy particulates was also investigated.

\section{Experimental Procedures}

Common commercial AZ91D magnesium alloy, the chemical composition of which is listed in Table 1, was chosen as the matrix material. Ti6Al4V particles (TC4p) with an average particle size of 15 and $35 \mu \mathrm{m}$ were employed as the reinforcements of the composites in the present study. The melt temperature-time curve representing the stir casting process is shown in Fig. 1 and the specific process flow diagram is shown in Fig. 2. $35 \mu \mathrm{m}$ 10 vol.\% TC4p/AZ91D magnesium matrix composites were selected as the example to investigate the effect of the casting parameters on the microstructure and mechanical properties of the composites. Five fabrication parameters are shown in Table 2. All samples of the composites and AZ91D alloy were prepared through the fabrication process based on Figs. 1 and 2 as follows. At first, AZ91D alloy was heated to $720^{\circ} \mathrm{C}$ in a mild steel crucible, which was in a resistance furnace with intelligent temperature controlling apparatus. At the same time, the protection atmosphere comprised of $\mathrm{CO}_{2}+\mathrm{SF}_{6}$ mixture was used to avoid burning of $\mathrm{Mg}$ during melting. The matrix alloy was then cooled down to the semisolid condition at $575{ }^{\circ} \mathrm{C}$ when it was completely melted. Next, the melt was stirred in the semisolid condition by stainless steel impeller to form a suitable vortex. Meanwhile, the Ti6Al4V particles preheated at $100{ }^{\circ} \mathrm{C}$ were added into the vortex of the melt during stirring. The aim of preheating TC4 particles is to eliminate the moisture on the surface of reinforcements. In addition, the preheating temperature could not be too high, and the TC4 particles would be seriously deteriorated due to excessive metal oxidation which might have resulted from extremely remarkable oxygen absorption capacity of titanium. After stirring in the semisolid condition for a given time as shown in Table 2, the composite melt was reheated to $700{ }^{\circ} \mathrm{C}$ and then kept at this temperature for $5 \mathrm{~min}$. Furthermore, the melt was still stirred during the reheating and the liquid-state composite holding process. Finally, the mixture melt was poured into a permanent steel mold to be solidified under a $100 \mathrm{MPa}$ pressure. It should be noted that the melt was invariably under the protection atmosphere.

The microstructures of the composites and AZ91D alloy were observed by using optical microscopy (OM; Olympus DP11), scanning electron microscopy (SEM; Quanta 200FEG) and transmission electron microscopy (TEM; Tecnai F30). All the samples for microstructure analysis were prepared by conventional mechanical polishing. Acetic picral $(5 \mathrm{ml}$ acetic acid $+5.5 \mathrm{~g}$ picric acid $+100 \mathrm{ml} \mathrm{H}_{2} \mathrm{O}+90 \mathrm{ml}$ ethanol) was used as etching reagent for the $\mathrm{OM}$ analysis. The TEM observation specimens were obtained by grinding-polishing and then

Table 1 Chemical composition of AZ91D magnesium alloy

\begin{tabular}{lllllllll}
\hline Al & $\mathrm{Zn}$ & $\mathrm{Mn}$ & $\mathrm{Si}$ & $\mathrm{Cu}$ & $\mathrm{Ni}$ & $\mathrm{Fe}$ & $\mathrm{Be}$ & $\mathrm{Mg}$ \\
\hline 9.29 & 0.71 & 0.23 & 0.019 & 0.001 & 0.001 & 0.0019 & 0.0014 & Balance \\
\hline
\end{tabular}




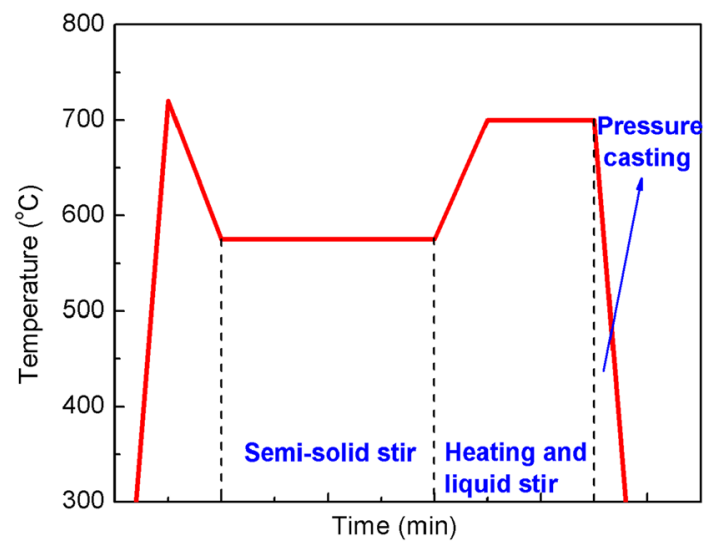

Fig. 1 Melt temperature-time curve for stir casting technological process

thinned by ion beam. The average grain sizes of the composites with different reinforcement volume fractions and different particle sizes were measured by Image ProPlus software based on the mean linear intercept method. The tensile tests were performed on an Instron Series 5569 testing machine at room temperature $\left(25^{\circ} \mathrm{C}\right)$ with the tensile rate of $0.5 \mathrm{~mm} / \mathrm{min}$ in accordance with ASTM: E8/E8M-13a standards. The average tensile data of each sample were obtained from five tests at the same condition to ensure the accuracy of the experimental data.

\section{Results and Discussion}

\subsection{Stir Casting Processing of the Composites}

The vortex stirring in semisolid condition is very efficient to break up particle clusters and disperse particles uniformly in the matrix. However, the vortex stirring for long time also leads to the pores and oxidation inclusions in the composites. Thus, the semisolid stir time plays a major role in the microstructure and mechanical properties of the composites. Figure 3 shows the microstructures of the composites fabricated by different semisolid stir time (ST1, ST-2 and ST-3). As shown in Fig. 3a, b, after semisolid stirring for $5 \mathrm{~min}$, the TC4p/AZ91 composites exhibited some particle-free regions and particle-rich regions, although particle agglomeration was eliminated. As the semisolid stir time increased to $10 \mathrm{~min}$, TC4 particle distribution was significantly improved. With the further increase in the semisolid stir time, the particle distribution was not further improved, but numerous oxidation inclusions as shown in Fig. 3e, f. Thus, by comparing ST-1, ST2 and ST-3, the particle distribution was the best in the composites stirred for $10 \mathrm{~min}$ in the semisolid state.

After semisolid stir, the heating stir speed should be slowed down in order to avoid introducing detrimental oxide inclusions into the composites, which is mainly due to the strong oxygen absorption ability of liquid

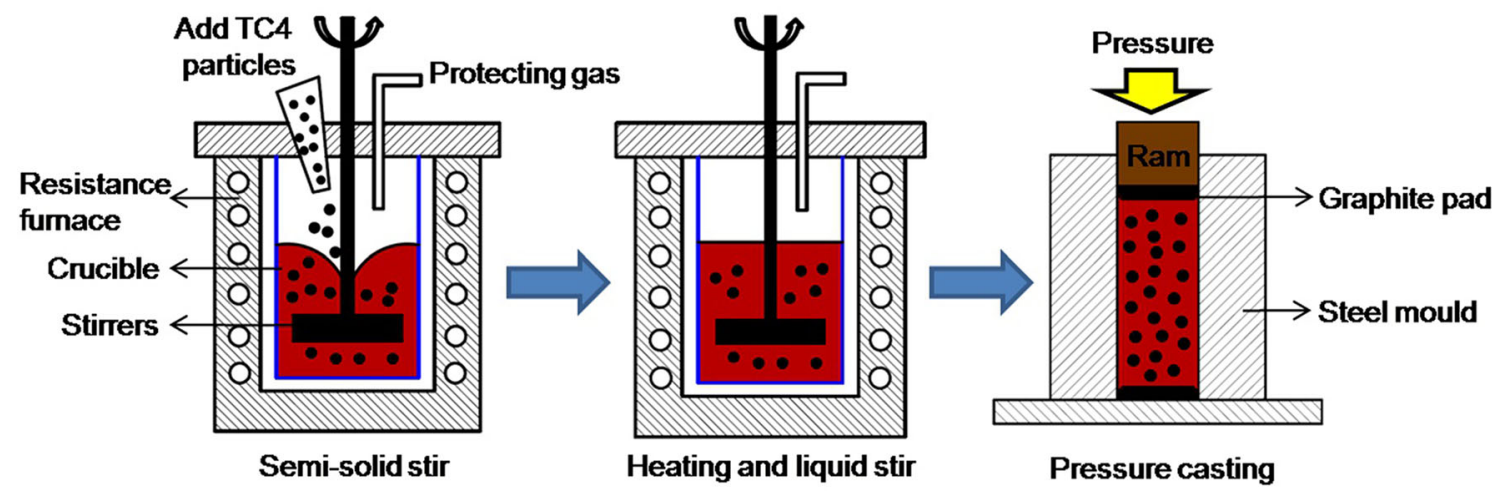

Fig. 2 Specific process flow diagram

Table 2 Different casting parameters for $35 \mu \mathrm{m} 10$ vol.\% TC4p/AZ91D composites

\begin{tabular}{|c|c|c|c|c|c|}
\hline \multirow[t]{2}{*}{ Experiment number } & \multicolumn{2}{|l|}{ Semisolid stir } & \multirow{2}{*}{$\begin{array}{l}\text { Heating stir } \\
\text { Speed (rpm) }\end{array}$} & \multicolumn{2}{|l|}{ Liquid stir } \\
\hline & Speed (rpm) & Time (min) & & Speed (rpm) & Time (min) \\
\hline ST-1 & 1000 & 5 & 300 & 250 & 5 \\
\hline ST-2 & 1000 & 10 & 300 & 250 & 5 \\
\hline ST-3 & 1000 & 15 & 300 & 250 & 5 \\
\hline ST-4 & 1000 & 10 & 600 & 250 & 5 \\
\hline
\end{tabular}



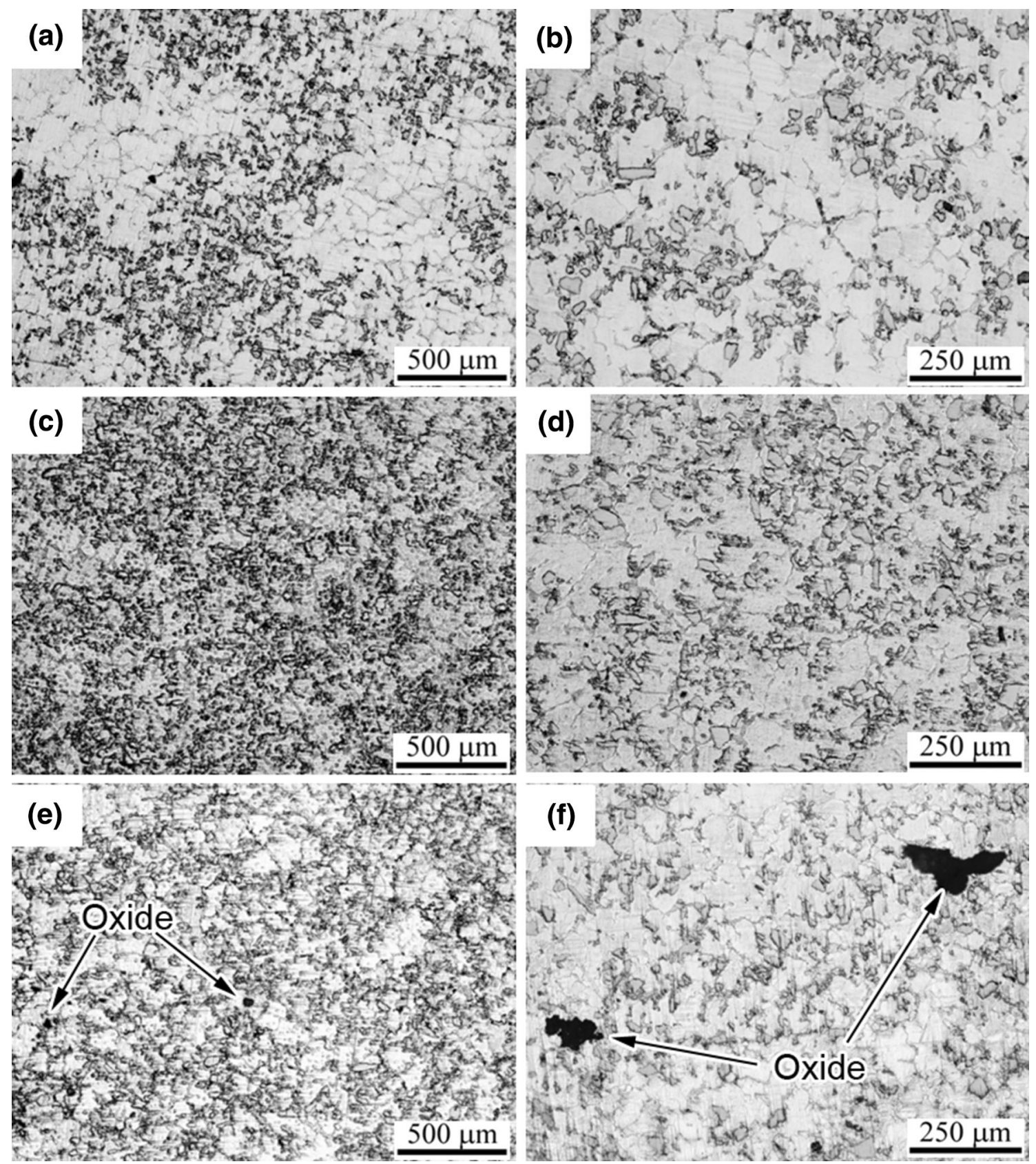

Fig. 3 OM images of the composites fabricated by different semisolid stir time: a, b $5 \mathrm{~min}, \mathbf{c}, \mathbf{d} 10 \mathrm{~min}, \mathbf{e}, \mathbf{f} 15 \mathrm{~min}$

magnesium. On the other hand, the heating stir needs to reach a certain speed for inhabiting the settling of TC4 particles caused by obvious density difference between TC4 particles and Mg matrix. Figure 4 shows the microstructures of the composites fabricated by different heating stir speeds (ST-2, ST-4). TC4 particles were distributed uniformly and no obvious particle clusters were observed in the composites fabricated by both ST-2 and ST-4, indicating that both ST-2 and ST-4 are fit for the TC4 particle distribution. In addition, no deposition of TC4 particles was observed at the bottom of the crucible when the mixture melt was poured into the metal mold during the process of ST-2 and ST-4. However, apparent oxide inclusions were observed in the composites when the heating stir speed was $600 \mathrm{rpm}$ as shown in Fig. 4c, d. Thus, the heating stir speed of $300 \mathrm{rpm}$ was better for not only overcoming settlement of particles but also avoiding oxide inclusions. Actually, we also fabricated the TC4p/ AZ91 composites by a lower heating stir speed of $200 \mathrm{rpm}$. Nevertheless, almost all the TC4 particles were deposited at the bottom of the crucible inside. It was indicated that the heating stir speed of $200 \mathrm{rpm}$ could not disperse the 

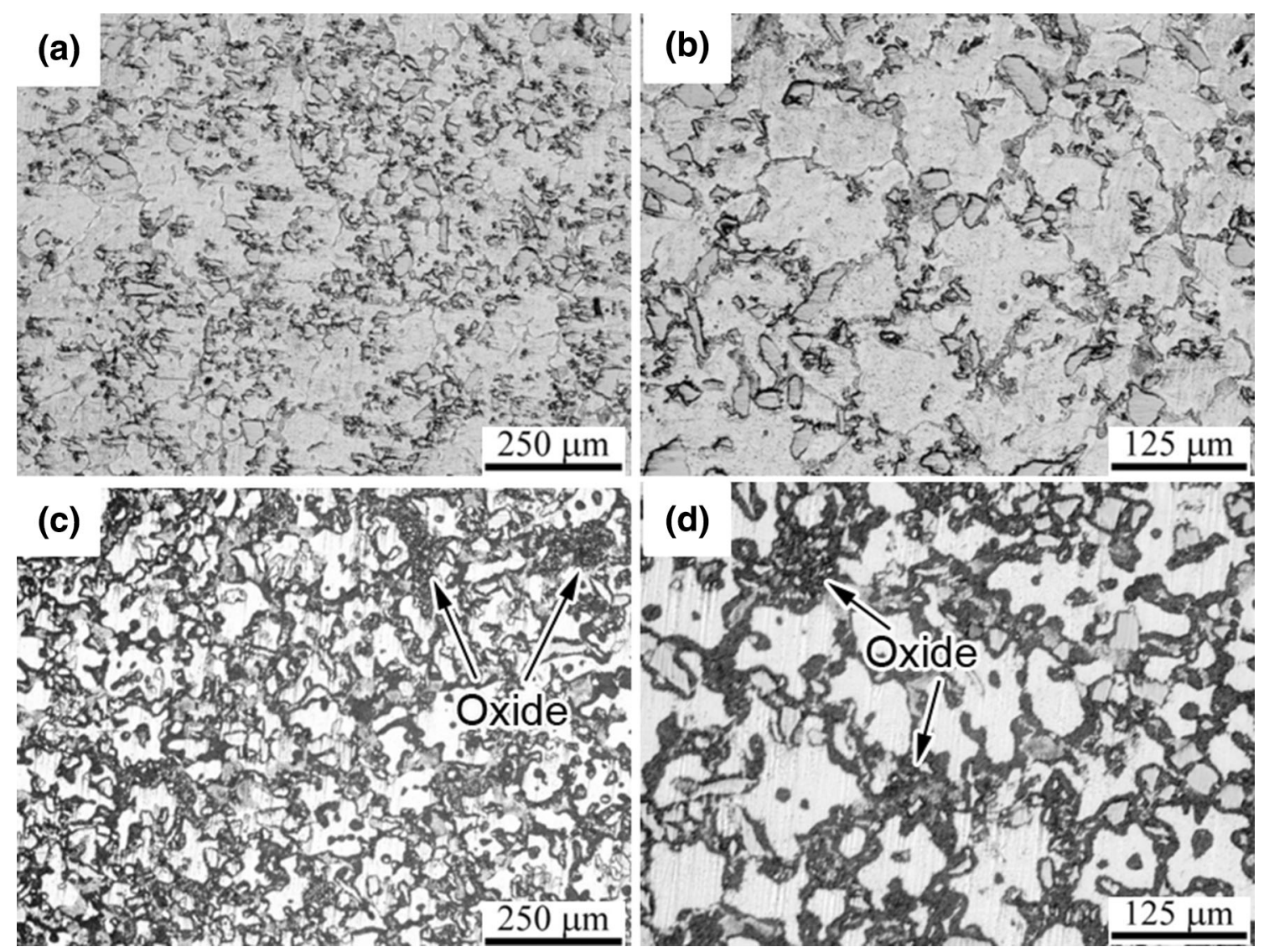

Fig. 4 OM images of the composites fabricated by different heating stir speeds: a, b $300 \mathrm{rpm}$, c, d $600 \mathrm{rpm}$

particles uniformly in the matrix and was undesirable to fabricate the TC4p/AZ91 composites.

The mechanical properties of the composites fabricated by different parameters are shown in Fig. 5 . The ultimate tensile strength (UTS) and elongation to fracture of the composites fabricated by ST-1 and ST-3 were clearly inferior to those of the composites stirred for $10 \mathrm{~min}$. The sharp decrease in tensile properties could be attributed to the degradation of the microstructure (particle distribution and oxidation inclusions) caused by the improper semisolid stir time. Under external tensile load, intense stress concentration can be developed around the particle enrichment regions in the microstructure of the composites stirred for $5 \mathrm{~min}$, and localized damage may occur when the local stress is beyond the ultimate fracture strength of the material. After semisolid stirring for $15 \mathrm{~min}$, voids and oxidation inclusions equivalent to preexisting cracks definitely cause an adverse effect on the mechanical properties of the composites. It can be seen that either too short or too long time for semisolid stirring is detrimental to the microstructure and the mechanical properties of the composites. Thus, $10 \mathrm{~min}$ is considered as the optimal time for semisolid stirring to fabricate $35 \mu \mathrm{m} 10$ vol.\% TC4p/AZ91 composites. By comparing ST-2 and ST-4, the TC4p/AZ91

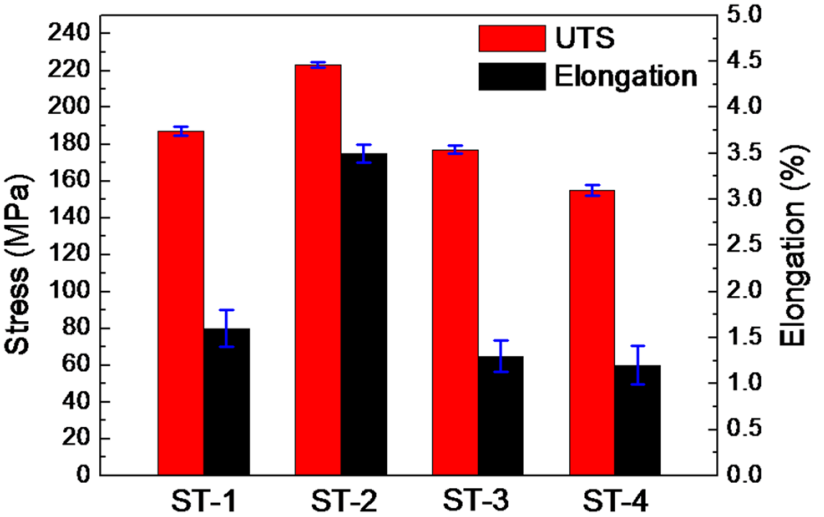

Fig. 5 Tensile properties of the composites fabricated by different casting parameters

composites employing the heating stir speed of $300 \mathrm{rpm}$ exhibited superior tensile properties. When higher stir speed in the heating state was employed, the defects containing pores and oxidation inclusions in the microstructure of the composites increased, implying more sites for the crack nucleation and therefore resulting in premature fracture of the composites. Thus, the heating stir speed of $300 \mathrm{rpm}$ is better for the mechanical properties of the composites. 

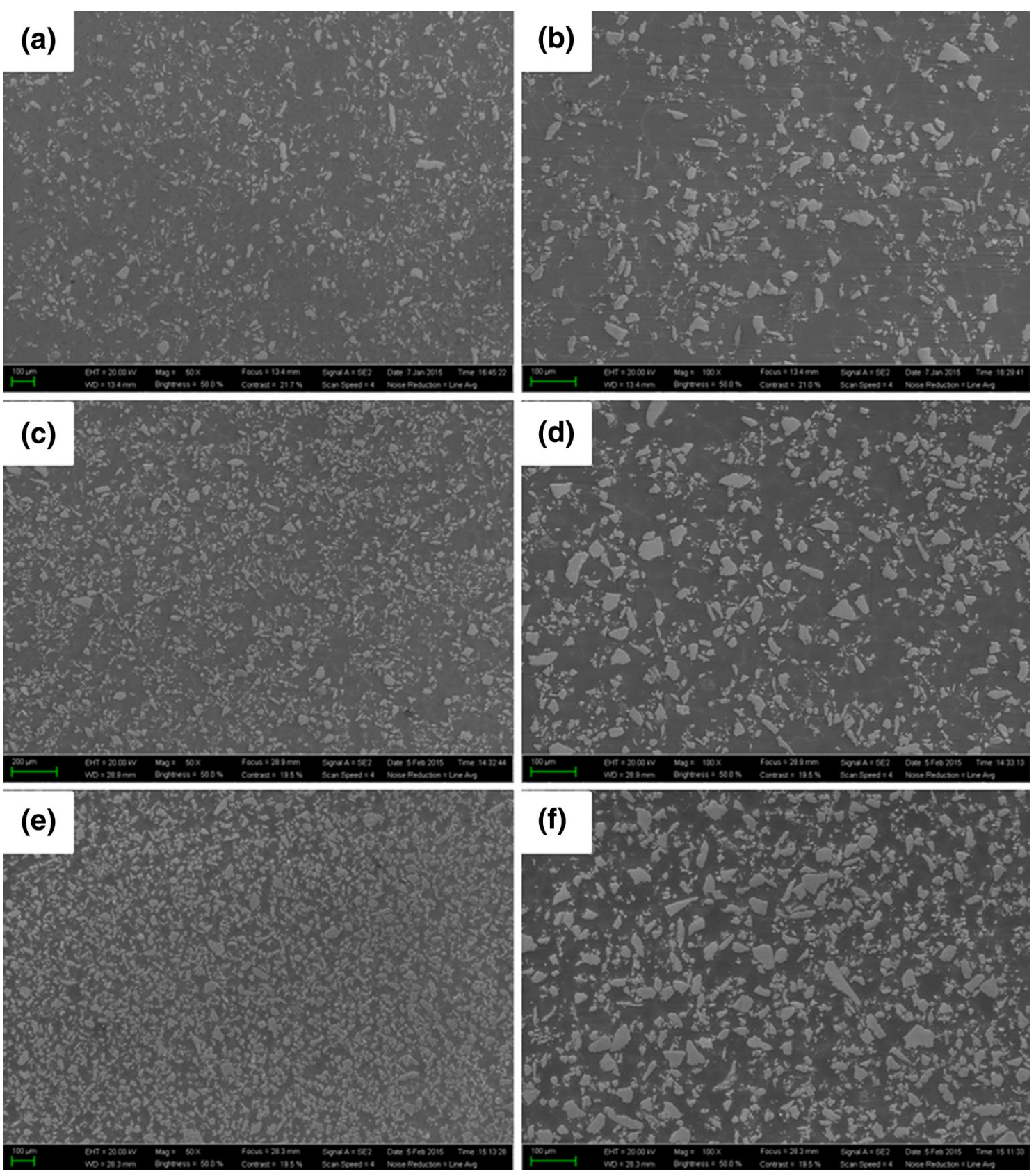

Fig. 6 SEM micrographs of the composites with different particle contents: a, b $10 \%$, c, d 15\%, e, f $20 \%$

\subsection{Microstructures of the Composites}

On the basis the above research results, we successfully obtained optimum technological parameters to fabricate the magnesium matrix composites with different volume fractions of TC4 particles (10, 15 and 20\%). The specific semisolid stir time for the composites with the particle volume fractions of 15 and $20 \%$ was 15 and $20 \mathrm{~min}$, respectively. Figure 6 presents the SEM micrographs of the composites with different particle contents. In these composites, TC4 particles distributed uniformly, and no evident oxidation inclusions were observed. Thus, the optimal parameters for the composites with different particle contents were obtained.

Figure 7 shows the OM images of AZ91 alloy and the composites with different particle volume fractions, and the average grain sizes of the composites are shown in Fig. 8. The grains in the composites were significantly refined due to the addition of TC4 particles. Furthermore, the average grain sizes of the composites decreased remarkably with the increase in the particle contents, which further proves the evident grain refinement of TC4 particles. The grain 

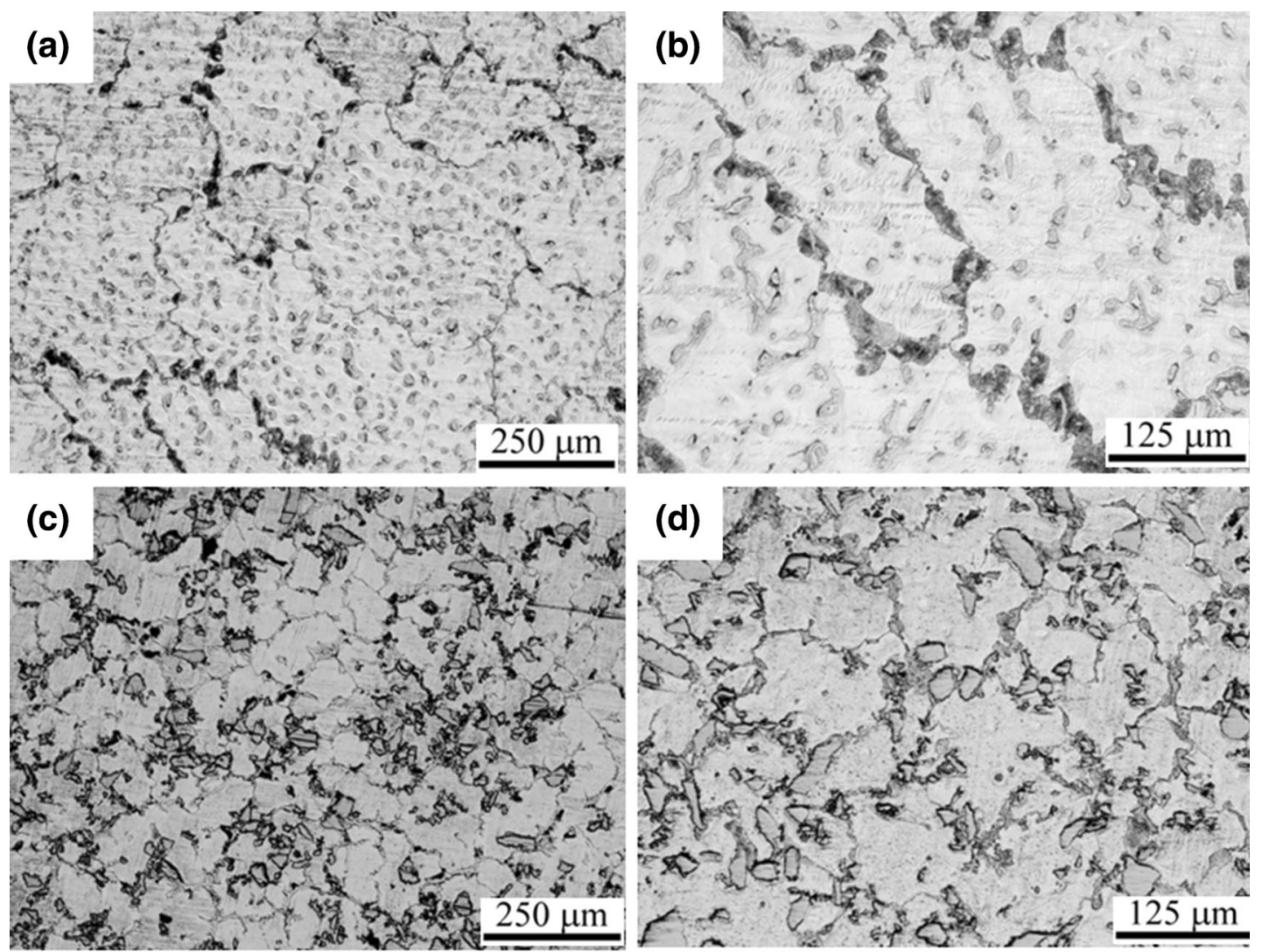

(e) 3 a s.t.

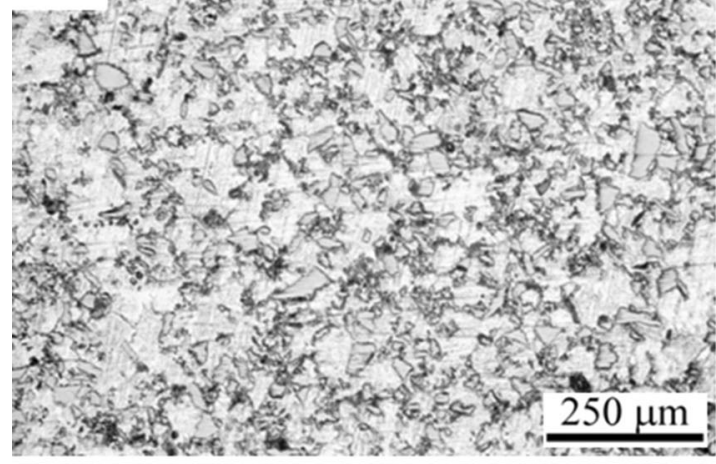

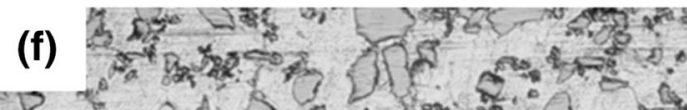
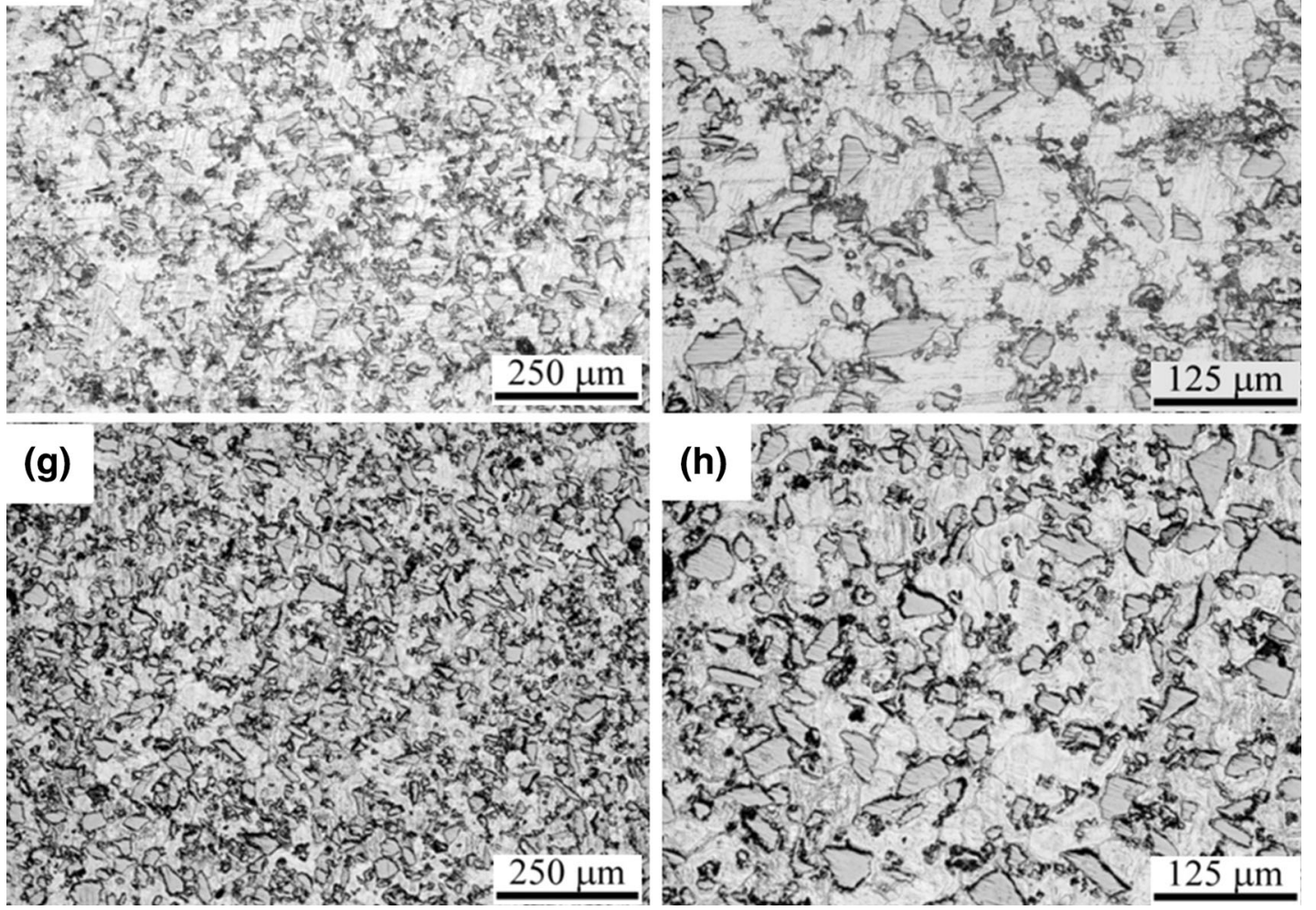

Fig. 7 OM images of AZ91 alloy and the composites with different particle volume fractions: a, b AZ91 alloy, c, d 10\%, e, f $15 \%$, g, h $20 \%$ 


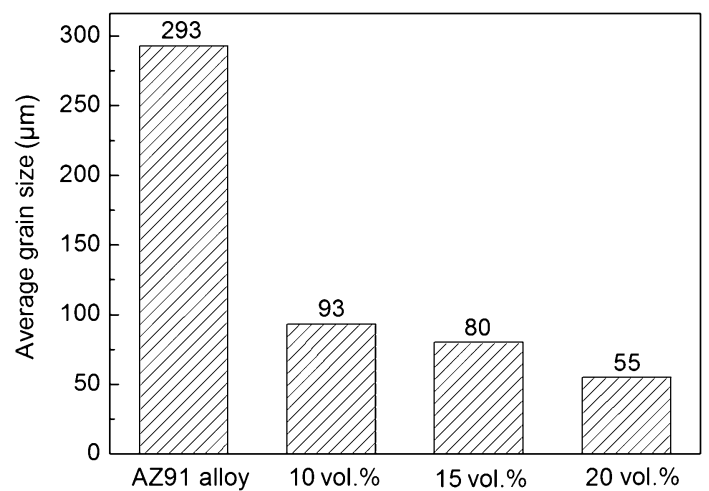

Fig. 8 Average grain sizes of AZ91 alloy and TC4p/AZ91 composites with different volume fractions

refinement mechanism of TC4 particles is mainly as follows: on the one hand, the surface of TC4 particles acting as heterogeneous nucleation sites can stimulate nucleation of the primary phase $\alpha-\mathrm{Mg}$ during the solidification process; on the other hand, the presence of hard TC4 particles can retard the growth of solidified $\mathrm{Mg}$ grains by constraining the grain boundary migration. As shown in Fig. 7a, b, coarse $\mathrm{Mg}_{17} \mathrm{Al}_{12}$ precipitations dispersed unevenly on the grain boundaries of AZ91 alloy, which certainly worsens the mechanical properties of the alloy. From Fig. 7d, f, h, some TC4 particles were segregated along the grain boundary regions resulting in relatively nonuniform "necklace-type" particle distribution [27], which is attributed to the "push" effect of the solid-liquid interface at the solidification front. Nevertheless, some particles distributed in the interior of grains since some of the TC4 particles could be captured by the freezing front due to the good wettability between TC4 particles and molten AZ91 alloy.

Figure 9 presents the element distribution near the TC4 particles in the $35 \mu \mathrm{m} 10 \mathrm{vol} \%$ TC4p/AZ91 composites. As shown in Fig. 9a, there were fine particle phases in a small quantity around TC4 particles. Some fine particles were attached on the surface of TC4 particles, and the others located in the matrix a little far from the big TC4 particles. There was no detectable trace of $\mathrm{Mg}$ element in the fine particle phases, indicating that the fine particles are not $\mathrm{Mg}_{17} \mathrm{Al}_{12}$, as shown in Fig. 9b. From Fig. 9c, d, Ti and $\mathrm{Al}$ are enriched at the matrix/particle interface and the fine particle phases, which confirm that the fine particles were $\mathrm{Al}-\mathrm{Ti}$ intermediate phases formed by the interfacial reaction.

Figure 10 shows the TEM micrograph of the interface between the TC4 particles and the matrix in the composites. Voids were not observed at the interface as shown in Fig. 10a. This indicates that the TC4 particles are bonded with the magnesium alloy matrix pretty well. In addition, there were some rod-like precipitate phases at the interface which extended from the TC4 particle to the AZ91 matrix. The rod-like precipitates may serve as a bridge between the reinforcements and matrix, so they might aid to interfacial integrity of TC4 particulates with the matrix. The rod-like precipitates may be beneficial to transfer the applied stress to $\mathrm{TC} 4$ particles. $\mathrm{Mg}$ does not react with $\mathrm{Ti}$, but $\mathrm{Al}$ in matrix alloy can react with $\mathrm{Ti}$ at the high temperature. Thus, $\mathrm{Al}$ atoms in the matrix and $\mathrm{Ti}$ atoms in the particles can form Al-Ti intermetallics at the interfaces. Yang et al. [28] have reported that the Gibbs free energies of formation of $\mathrm{Al}_{3} \mathrm{Ti}, \mathrm{Al}_{2} \mathrm{Ti}$ and $\mathrm{AlTi}_{3}$ were $-33,-36$ and $-25 \mathrm{~kJ} / \mathrm{mol}$ under the condition of $700{ }^{\circ} \mathrm{C}$, respectively. Besides, $\mathrm{Al}_{2} \mathrm{Ti}$ was reported to appear as the transitional phases only when AlTi stably exists [29]. It was also found that Al-Ti intermetallics had a lower standard enthalpy of formation than $\mathrm{Mg}-\mathrm{Al}$ intermediate phases, indicating that $\mathrm{Al}$ has a stronger affinity with Ti. Thus, the rod-like precipitates might be supposed to be $\mathrm{Al}_{3} \mathrm{Ti}$ in the present study. The selected area electron diffraction (SAED) pattern from the rod-like precipitates is shown in Fig. 10b, confirming that they were $\mathrm{Al}_{3}$ Ti. Sankaranarayanan and Jayalakshmi [24] also found the rod-like $\mathrm{Al}_{3} \mathrm{Ti}$ intermetallic phases in the process of synthesizing pure magnesium incorporated with $\mathrm{Al}$ and $\mathrm{Ti}$ elements. As pointed with black arrows in Fig. 10, some $\mathrm{Al}_{3} \mathrm{Ti}$ precipitates located in the matrix but not on the surface of the particles, which were likely due to fracture of $\mathrm{Al}_{3} \mathrm{Ti}$ precipitates during stirring. They are like short fiber reinforcements in the matrix to strengthen the composites further.

\subsection{Mechanical Properties of the Composites}

Figure 11 shows the mechanical properties of the composites with different volume fractions of the particles. As shown in Fig. 11a, the addition of TC4 particles evidently improved the yield strength (YS) and ultimate tensile strength (UTS). What is more, with the increase in the volume fraction of TC4 particles, the YS and UTS of the composites were significantly enhanced. The remarkable increase in the strengths of the composites depends on the following aspects. Firstly, the average grain size in the matrix decreased with increasing particle contents as shown in Fig. 8, which could improve the YS of the composites according to the Hall-Petch equation [30]. Secondly, the difference thermal expansion coefficient between TC4 particles and AZ91 matrix generated thermal residual stresses around the reinforcements during solidification, producing high-density dislocations to store in the matrix near the particles. The increase in the YS ascribed to the thermal mismatch between TC4p and AZ91 alloy $\left(\Delta \sigma_{\mathrm{CET}}\right)$ can be expressed as [31]:

$\Delta \sigma_{\mathrm{CTE}}=3^{1 / 2} \beta \mu_{\mathrm{m}} b\{12 f \Delta \alpha \Delta T /((1-f) b d)\}^{1 / 2}$, 

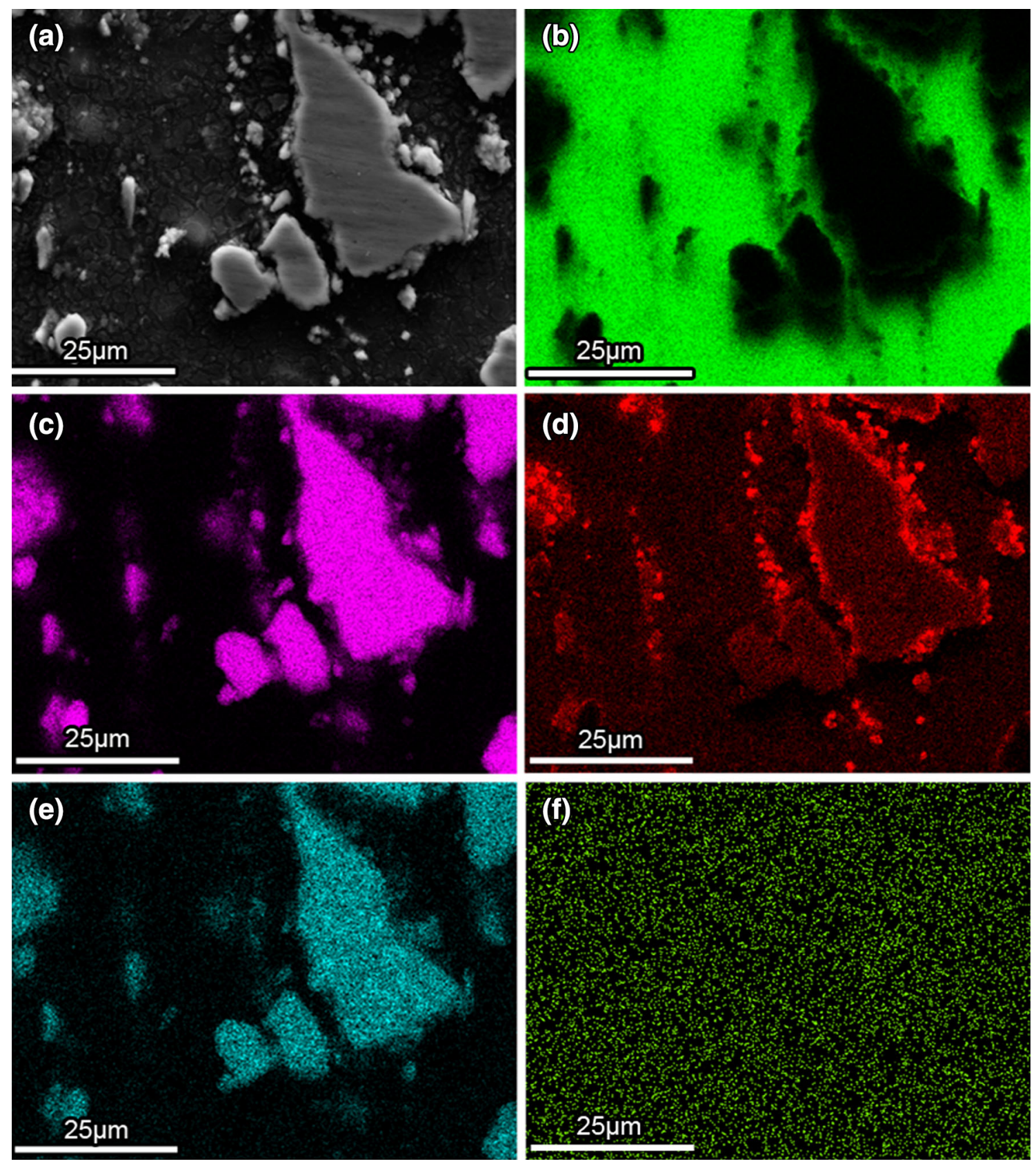

Fig. 9 Element distribution near the TC4 particles in the $35 \mu \mathrm{m} 10$ vol.\% TC4p/AZ91 composites: a SEM image around the TC4 particles, b $\mathrm{Mg}, \mathbf{c} \mathrm{Ti}, \mathbf{d} \mathrm{Al}, \mathbf{e ~ V}, \mathbf{f} \mathrm{Zn}$

where $\beta$ is the strengthening constant, $\mu_{\mathrm{m}}$ the shear modulus of the matrix, $b$ is Burgers vector, $f$ is the volume fraction of the particles, $\Delta \alpha$ is the difference of coefficient of thermal expansion (CTE) between AZ91 alloy and TC4p, $\Delta T$ is the difference between solidification temperature and room temperature, $d$ is the average size of TC4p. With increasing addition of TC4 particles, the dislocation density is gradually heightened, which can further improve the YS of the composites. Thirdly, the applied stress could be efficiently transferred to the TC4 particles as a result of good interface bonding. Furthermore, the increase in the particle contents could simultaneously strengthen the effect on the load transfer. Fourthly, coarse $\mathrm{Mg}_{17} \mathrm{Al}_{12}$ precipitations continuously dispersed along the grain boundaries of $\alpha-\mathrm{Mg}$ in AZ91 alloy as shown in Fig. 7b. In such case, the tensile strength would be decreased $[32,33]$. However, the presence of TC4p improved the distribution of $\mathrm{Mg}_{17} \mathrm{Al}_{12}$ precipitations as shown in Fig. $7 \mathrm{~d}, \mathrm{f}, \mathrm{h}$, resulting in the second phases more finer and distributed more evenly in the matrix. In addition to the above reasons, the homogeneous particle distribution arising from a reasonable processing technology and better wettability between AZ91 


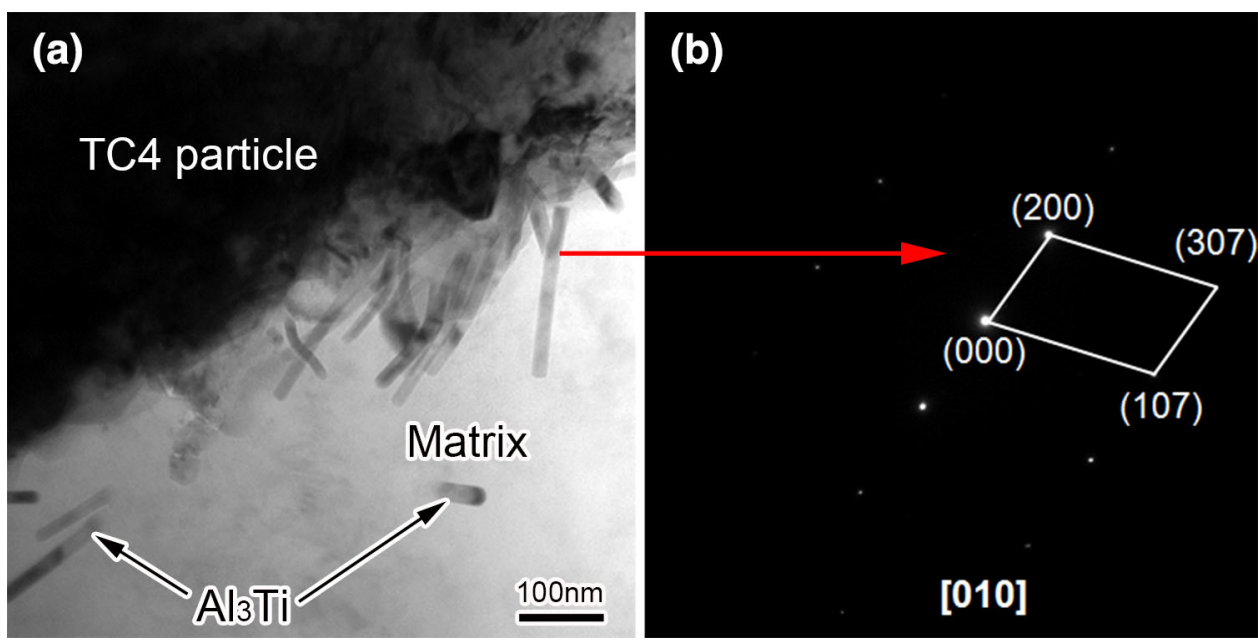

Fig. 10 TEM micrograph of the interface between the TC4 particles and the matrix in the composites: a the morphology of the interface, $\mathbf{b}$ the SAED pattern of $\mathrm{Al}_{3} \mathrm{Ti}$
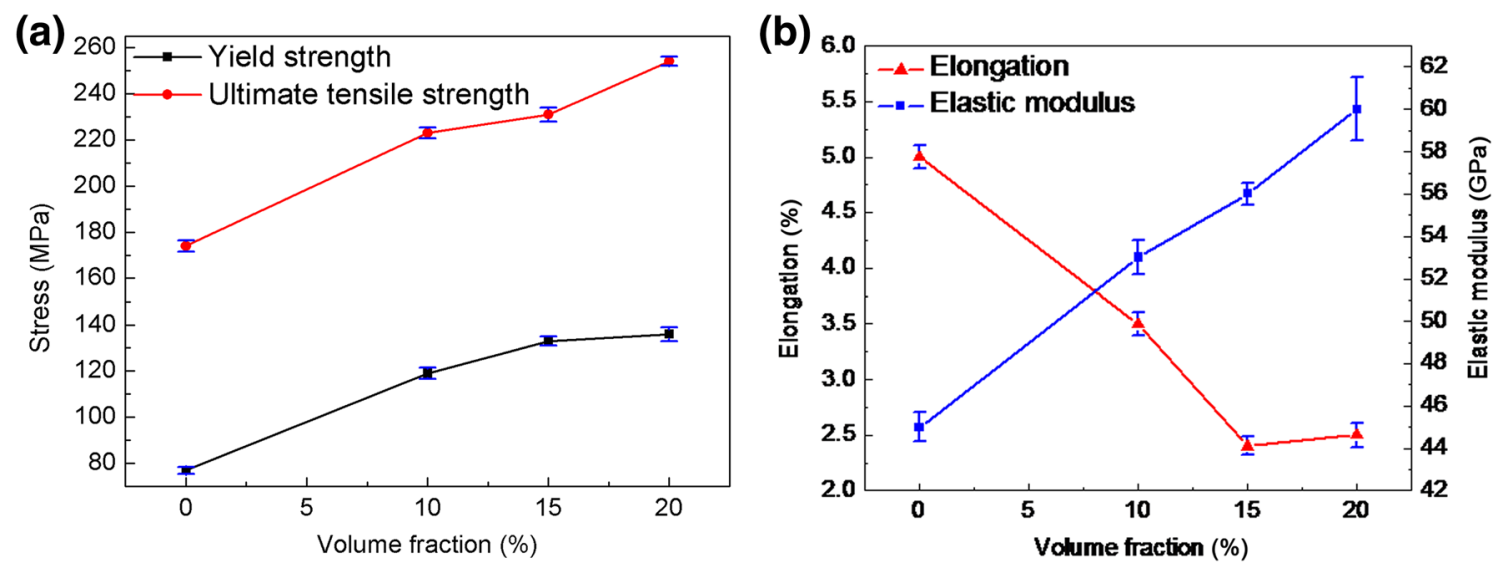

Fig. 11 Mechanical properties of the composites with different particle volume fractions: a YS and UTS, b elongation and elastic modulus

alloy and TC4p also plays a major role in increasing the strengths of the composites. It is worth mentioning that the TC4p/AZ91 composites had a significant advantage over the SiCp/AZ91 composites with equal particle contents in UTS [26, 33, 34]. As an example, the UTS of 20 vol.\% TC4p/AZ91 composites reached $254 \mathrm{MPa}$, however, the UTS of SiCp/AZ91 composites with $20 \%$ volume fraction particles was $<200 \mathrm{MPa}$ [26].

From Fig. 11b, it can be seen that increasing addition of TC4 particles improved the elastic modulus of the composites. However, the elongations were reduced. According to the reported studies $[26,33,34]$, the elongation of the composites in the present study was much higher than that of the same matrix composites reinforced with $\mathrm{SiC}$ particles fabricated by the similar methods, whose elongations are approximately $<2 \%$ so far. The higher elongation of the TC4p/AZ91 composites is attributed to the following reasons. First of all, superior wettability between TC4 particle and magnesium alloy contributes to not only the good interface bonding between the reinforcements and the matrix resulting in more efficient load transfer, but also enables some TC4 particles to be swallowed by the freezing front in the solidification instead of only being pushed to grain boundaries. Thus, the particle distribution was more homogeneous in the present composites. Next, relatively small difference in thermal expansion coefficient and elastic modulus between $\mathrm{Ti}$ and $\mathrm{Mg}$ induces lower residual stresses in the composites. In the case, microcracks would not be generated and developed prematurely at the interfaces so that the elongation of the composites is almost retained. Last but not least, TC4 particles and magnesium matrix in the composites might be deformed together by means of coordinating with each other, which is due to the additional benefit that TC4 particles can deform plastically. Hence, under external load, the cracks at the interfaces could take place more uneasily in the TC4p/AZ91 composites, which gives rise to better toughness of the composites. 


\section{Conclusion}

The novel TC4p/AZ91 composites were successfully fabricated by stir casting. Through optimizing the stir casting parameters, TC4 particles could be dispersed uniformly in the matrix and oxidation inclusions were not observed in the composites with different particle volume fractions. The grains in the composites were significantly refined compared with AZ91 alloy. The TC4 particles bonded pretty well with the matrix. There were some $\mathrm{Al}_{3} \mathrm{Ti}$ phases around the particles formed by the interfacial reaction. The rod-like $\mathrm{Al}_{3} \mathrm{Ti}$ precipitates extending from the TC4 particle to the alloy matrix might improve the interfacial integrity. The addition of TC4 particles evidently improved the YS, UTS and elastic modulus. In addition, the elongation of the TC4p/AZ91 composites was much higher than those of the same matrix composites reinforced with $\mathrm{SiC}$ particles fabricated by stir casting.

Acknowledgments This work was supported by the National Natural Science Foundation of China (Grant No. 51471059) and the China Postdoctoral Science Foundation (Grant No. 2014T70328).

\section{References}

[1] R. Wu, Y. Yan, G. Wang, L.E. Murr, W. Han, Z. Zhang, M. Zhang, Int. Mater. Rev. 60, 65 (2014)

[2] A. Das, S.P. Harimkar, J. Mater. Sci. Technol. 30, 1059 (2014)

[3] Z. Yang, J.P. Li, J.X. Zhang, G.W. Lorimer, J. Robson, Acta Metall. Sin. (Engl. Lett.) 21, 313 (2008)

[4] Z.M. Zhang, C.J. Xu, X.F. Guo, S.Z. Jia, Acta Metall. Sin. (Engl. Lett.) 21, 177 (2008)

[5] Y.T. Yao, L.Q. Chen, J. Mater. Sci. Technol. 30, 661 (2014)

[6] K. Su, K.K. Deng, F.J. Xu, K.B. Nie, L. Zhang, X. Zhang, W.J. Li, Acta Metall. Sin. (Engl. Lett.) 28, 1015 (2015)

[7] H.Z. Ye, X.Y. Liu, J. Alloys Compd. 402, 162 (2005)

[8] P. Pérez, G. Garcés, P. Adeva, Compos. Sci. Technol. 64, 145 (2004)

[9] P. Poddar, V.C. Srivastava, P.K. De, K.L. Sahoo, Mater. Sci. Eng. A 460-461, 357 (2007)
[10] L. Chen, Y. Yao, Acta Metall. Sin. (Engl. Lett.) 27, 762 (2014)

[11] J. Umeda, M. Kawakami, K. Kondoh, E.L.S. Ayman, H. Imai, Mater. Chem. Phys. 123, 649 (2010)

[12] X. Zhang, Q. Zhang, H. Hu, Mater. Sci. Eng. A 607, 269 (2014)

[13] M.J. Shen, X.J. Wang, C.D. Li, M.F. Zhang, X.S. Hu, M.Y. Zheng, K. Wu, Mater. Des. 54, 436 (2014)

[14] Y.L. Xi, D.L. Chai, W.X. Zhang, J.E. Zhou, Scr. Mater. 54, 19 (2006)

[15] K. Kondoh, M. Kawakami, H. Imai, J. Umeda, H. Fujii, Acta Mater. 58, 606 (2010)

[16] Y.L. Xi, D.L. Chai, W.X. Zhang, J.E. Zhou, Mater. Lett. 59, 1831 (2005)

[17] M. Rashad, F. Pan, M. Asif, J. She, A. Ullah, J. Magnes. Alloys 3, 1 (2015)

[18] S. Sankaranarayanan, S. Jayalakshmi, M. Gupta, J. Alloys Compd. 509, 7229 (2011)

[19] M. Rashad, F. Pan, A. Tang, Y. Lu, M. Asif, S. Hussain, J. She, J. Gou, J. Mao, J. Magnes. Alloys 1, 242 (2013)

[20] S. Sankaranarayanan, S. Jayalakshmi, M. Gupta, Mater. Des. 37, 274 (2012)

[21] S. Sankaranarayanan, S. Jayalakshmi, M. Gupta, J. Magnes. Alloys 2, 13 (2014)

[22] G.K. Meenashisundaram, M. Gupta, J. Alloys Compd. 593, 176 (2014)

[23] S.F. Hassan, M. Gupta, J. Alloys Compd. 345, 246 (2002)

[24] S. Sankaranarayanan, S. Jayalakshmi, M. Gupta, Mater. Sci. Eng. A 530, 149 (2011)

[25] S. Sankaranarayanan, R.K. Sabat, S. Jayalakshmi, S. Suwas, M. Gupta, J. Alloys Compd. 575, 207 (2013)

[26] X.J. Wang, N.Z. Wang, L.Y. Wang, X.S. Hu, K. Wu, Y.Q. Wang, Y.D. Huang, Mater. Des. 57, 638 (2014)

[27] X.J. Wang, X.S. Hu, K.B. Nie, K.K. Deng, K. Wu, M.Y. Zheng, Mater. Sci. Eng. A 545, 38 (2012)

[28] Z.R. Yang, S.Q. Wang, M.J. Gao, Y.T. Zhao, K.M. Chen, X.H. Cui, Compos. Pt. A Appl. Sci. Manuf. 39, 1427 (2008)

[29] L.M. Peng, H. Li, J.H. Wang, Mater. Sci. Eng. A 406, 309 (2005)

[30] K.K. Deng, X.J. Wang, M.Y. Zheng, K. Wu, Mater. Sci. Eng. A 560, 824 (2013)

[31] C. Goh, J. Wei, L. Lee, M. Gupta, Acta Mater. 55, 5115 (2007)

[32] W.M. Gan, K. Wu, M.Y. Zheng, X.J. Wang, H. Chang, H.G. Brokmeier, Mater. Sci. Eng. A 516, 283 (2009)

[33] K.B. Nie, X.J. Wang, K. Wu, X.S. Hu, M.Y. Zheng, L. Xu, Mater. Sci. Eng. A 528, 8709 (2011)

[34] S. Aravindan, P.V. Rao, K. Ponappa, J. Magnes. Alloys 3, 52 (2015) 